\title{
Evaluation of the Estrogenic Activity of Pueraria (Kudzu) Flower Extract and Its Major Isoflavones Using ER-Binding and Uterotrophic Bioassays
}

\author{
Tomoyasu Kamiya $^{1 *}$, Akira Takano ${ }^{1}$, Yayoi Kido ${ }^{1}$, Yuki Matsuzuka ${ }^{1}$, Mayu Sameshima-Kamiya ${ }^{1}$, \\ Masahito Tsubata $^{1}$, Motoya Ikeguchi ${ }^{1}$, Kinya Takagaki ${ }^{1}$, Junei Kinjo ${ }^{2}$ \\ ${ }^{1}$ Research and Development Division, Toyoshinyaku Co. Ltd., Tosu-shi, Japan; ${ }^{2}$ Faculty of Pharmaceutical Sciences, Fukuoka Uni- \\ versity, Fukuoka, Japan. \\ Email: kamiyat@toyoshinyaku.co.jp
}

Received January $20^{\text {th }}, 2013$; revised March $5^{\text {th }}, 2013$; accepted April $14^{\text {th }}, 2013$

Copyright (C) 2013 Tomoyasu Kamiya et al. This is an open access article distributed under the Creative Commons Attribution License, which permits unrestricted use, distribution, and reproduction in any medium, provided the original work is properly cited.

\begin{abstract}
Pueraria flower extract (PFE) is a hot water extract of the Kudzu flower (Pueraria thomsonii). Tea made from dried Kudzu flower is widely used in China, and PFE is utilized as a nutritional supplement in Japan. PFE contains unique isoflavones such as 6-hydroxygenistein 6,7-di- $O$-glucoside (6HGDG), tectorigenin 7-O-xylosylglucoside (TGXG), and tectoridin. 6HGDG is known to be metabolized into 6-hydroxygenistein, and TGXG and tectoridin are known to be metabolized into tectorigenin in the digestive tract. Isoflavones typically mimic the effects $\beta$-estradiol has on estrogen receptors (ERs) and may influence the female genital system in the case of excessive intake. As a result, the upper limit of safe daily consumption of soy isoflavones has been enforced in Japan. In the present study, ER-binding assays were performed using the EnBio estrogen receptor/cofactor assay system to compare the estrogenic activity of 6-hydroxygenistein and tectorigenin to that of the soy isoflavone genistein. In addition, uterotrophic bioassays were performed to investigate the estrogenic effects of PFE in vivo. The ER-binding assays revealed that the ER-binding affinities of 6-hydroxygenistein and tectorigenin were approximately $0.01-0.04$ that of genistein. Soy isoflavone products also induced an increase in uterine wet and blotted weight at doses of $500 \mathrm{mg} / \mathrm{kg}$ and $1000 \mathrm{mg} / \mathrm{kg}$, whereas PFE did not cause adverse estrogenic effects, even at a dose of $1000 \mathrm{mg} / \mathrm{kg}$. Based on these results, PFE does not appear to contain compounds with strong estrogenic activity or cause adverse estrogenic effects in vivo. Importantly, the results of this study confirm the safety of PFE as a food supplement.
\end{abstract}

Keywords: Estrogenic Activity; 6-Hydroxygenistein; Kudzu Flower Extract; Safety Evaluation; Tectorigenin

\section{Introduction}

Pueraria flower extract (PFE) is a hot water extract of the Kudzu flower (Pueraria thomsonii). Kudzu is a leguminous plant that grows in Japan, China, and other countries. The Kudzu flower is frequently used in traditional Chinese medicine for counteracting symptoms associated with alcohol use, liver injury, and menopause. In China, tea made from dried Kudzu flowers is widely consumed [1], and PFE is consumed as a nutritional supplement for the treatment of hangovers and obesity in Japan. We previously reported that PFE exerts anti-obesity effects in humans and mice fed high-fat diets [2-4]. In addition, we also reported that the isoflavone-rich

"Corresponding author. fraction of dietary PFE exhibits anti-obesity effect in mice fed high-fat diets [5].

The following isoflavones are found in the Kudzu flower but not in soy: 6-hydroxygenistein 6,7-di- $O$-glucoside (6HGDG), tectorigenin 7-O-xylosylglucoside (TGXG), tectoridin, genistin, glycitin, tectorigenin, genistein, and glycitein [5]. Among these isoflavones, 6HGDG, TGXG, tectoridin and tectorigenin, which comprise nearly $90 \%$ of the isoflavones in the Kudzu flower, are present in PFE. Previous studies have shown that the hydrolysis of the sugar moiety is required for isoflavone bioavailability and that isoflavone glycosides are not absorbed intact across the enterocyte in healthy adults [6]. When cultured with enteric bacteria, tectoridin and TGXG are metabolized into tectorigenin and 6HGDG is metabolized into 
the aglycone isoflavone 6-hydroxygenistein [7,8]. Consequently, the major isoflavones in PFE are likely to be absorbed into the bloodstream in the forms of tectorigenin and 6-hydroxygenistein.

Isoflavones are polyphenolic compounds that are capable of exerting estrogen-like effects. For this reason, isoflavones are classified as phytoestrogens. Due to their conformational similarity to $\beta$-estradiol, isoflavones exbihit similar effects on estorogen receptors (ERs). Phytoestrogens have recently received great attention because of their beneficial effects, including the prevention of atherosclerosis [9], and bone density loss [10]. However, the consumption of relatively high levels of some phytoestrogens has been shown to increase the risks of breast cancer [11], endometrial hyperplasia [12], and reproductive toxicity [13]. A previous study demonstrated that the high binding affinities of select isoflavones is associated with the structural properties of these molecules. For example, the binding affinities of select molecules for ER- $\alpha$ and $-\beta$ were observed in the following order: genistein $>$ daidzein $>$ biochanin $\mathrm{A}>$ glycitein $>$ formononetin A (ER- $\alpha)$; and genistein $>$ glycitein $>$ daidzein $>$ formononetine $>$ biochanin A $($ ER- $\beta)$ [14]. Based on these results, soy isoflavones, especially genistein are thought to exhibit strong estrogenic activity. In 2006, the Food Safety Commission of Japan announced their recommendations concerning the consumption of soy isoflavones, and the upper limit of safe daily consumption of soy isoflavones was set to a total of 75 $\mathrm{mg} /$ day as aglycones and $30 \mathrm{mg} /$ day as supplements in Japan.

For these reasons, the estrogenic activity of isoflavones commonly in foods should be investigated. Especially, to obtain the relative estrogenic activities against soy isoflavone is thought to be valuable. PFE is widely used in China and Japan, but there are no reports about the relative estrogenic activity of PFE in vivo. ER-binding assays are frequently used to compare the estrogenicity of compounds (Zacharewski, 1998), and uterotrophic bioassays are in vivo screening assays used to evaluate the ability of a chemical to elicit estrogenic activities consistent with agonists or antagonists of natural estrogens (e.g., $\beta$-estradiol). In this study, we conducted ERbinding assays to investigate the relative estrogenic activities of 6-hydroxygenistein and tectorigenin, the aglycone forms of PFE's major isoflavones as well as uterotrophic bioassays to investigate the estrogenic effects of PFE in vivo.

\section{Materials and Methods}

\subsection{Experimental Materials}

PFE was purchased from Ohta's Isan Co. Ltd. (Ibaraki,
Japan). PFE contains TGXG (8.5\%), tectoridin (4.6\%), 6HGDG $(3.4 \%)$, tectorigenin $(0.9 \%)$, genistin $(0.3 \%)$, glycitin $(0.2 \%)$, glycitein $(0.1 \%)$, and genistein $(0.1 \%)$. Quantitative analysis methods of isoflavones in PFE have been reported by Kamiya T. et al. [5]. Soy isoflavone product (SOY) was obtained from Nippon Bulk Yakuhin Co., Ltd. (Osaka, Japan). SOY contains $42.7 \%$ isoflavones (daidzein, daidzin, and a daidzin derivative, 23.3\%; genistein, genistin, and a genistin derivative, 5.6\%; glycitein, glycitin, and a glycitin derivative, $13.2 \%$ ). The dietary soy isoflavone-aglycone (aglycone equivalent) testing method (see Food Safety Notification No. 0823001, August 23, 2006 for guidelines regarding the handling of select health foods, including soy isoflavones) was used to perform a quantitative analysis of isofla- vones present in SOY. Tectorigenin, 6-hydroxygenistein, and genistein were purchased from Tokiwa Phytochemi- cal Co., Ltd. (Chiba, Japan). Equol was purchased from ChromoDex, Inc (California, USA), and ethinylestradiol was purchased from Tokyo Chemical Industry Co., Ltd. (Tokyo, Japan). Figure 1 shows the chemical structures of Kudzu isoflavones.

\subsection{ER-Binding Assays}

The binding affinity of phytoestrogens to ER- $\alpha$ and $-\beta$ was measured using the EnBio estrogen receptor cofactor assay system according to the manufacturer's protocol (EnBio Tec Laboratories, Japan). Briefly, we added biotinylated coactivator peptide to an avidin-coated plate and incubated the plate for $1 \mathrm{~h}$ at room temperature. We mixed estrogenic compounds, such as $\beta$-estradiol (provided by the manufacturer), tectorigenin, 6-hydroxygenistein, genistein, and equol with recombinant human ER$\alpha$ and $-\beta$. Then, the mixtures of ER- $\alpha$ and $-\beta$ were added to the biotinylated coactivator-coated plates, which were subsequently incubated for $1 \mathrm{~h}$ at room temperature. After washing the plate three times with the wash buffer provided by the manufacturer, anti-ER- $\alpha$ and $-\beta$ antibodies linked to HRP were applied to each well. After incu-

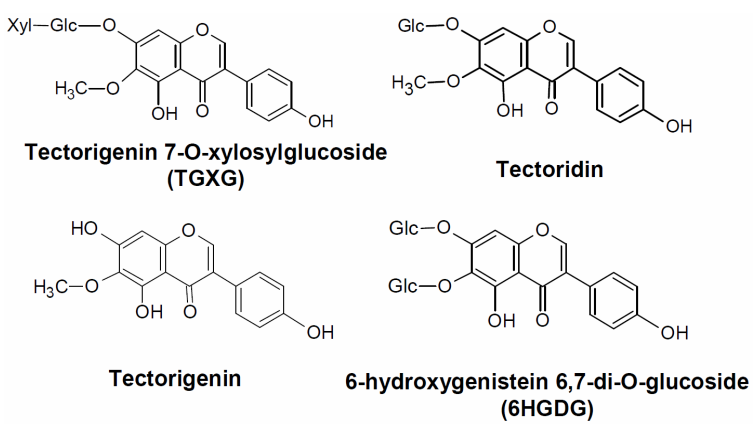

Figure 1. Chemical structures of tectorigenin 7-O-xylosylglucoside (TGXG), tectoridin, tectorigenin, and 6-hydroxygenistein 6,7-di- $O$-glucoside (6HGDG). 
bating the mixture for $30 \mathrm{~min}$ at room temperature, the plate was washed three times and a 3,3',5,5'-tetramethylbenzidine substrate solution was added. The absorbance of each well was measured at $450 \mathrm{~nm}$ using a Powerscan HT (DS Pharma Biomedical Co., Ltd., Japan).

\subsection{Uterotrophic Bioassays}

Uterotrophic bioassays were performed in accordance with the OECD Guidelines for the Testing of Chemicals (Uterotrophic bioassays in rodents. a short-term screening test for oestrogenic properties; No. 440, adopted on October 16, 2007). All procedures involving animals were performed in accordance with the guidelines for the animal experiments at CERI Hita according to Laboratory Animal Science (1987) by the American Association for Laboratory Animal Science and were approved by the Ethical Committee of the Chemicals Evaluation and Research Institute (Oita, Japan). Briefly, female SpragueDawley rats ovariectomized at the age of 6 weeks were purchased from Japan SLC Inc. (Sizuoka, Japan) at the age of 7 weeks. After being acclimated for 1 week, each Sprague-Dawley rat was weighed and assigned to one of eight groups in order for each group to have a uniform mean body weight. Rats were given daily po injections of either purified water (vehicle control), PFE $(250,500$, and $1000 \mathrm{mg} / \mathrm{kg}$ ), SOY $(250,500$, and $1000 \mathrm{mg} / \mathrm{kg}$ ), or s.c. injections of ethinylestradiol (EE, $0.6 \mu \mathrm{g} / \mathrm{kg}$; positive control) for 7 days. At the end of treatment, the rats were sacrificed, and each uterus and vagina was removed. The wet and blotted uterus weights were measured, and macroscopic examinations of the uterus and vagina were performed.

Data were expressed as the mean \pm SD. The body and uterus weights were initially analyzed using Bartlett's test of homogeneity of variance. When the results were determined to not be significant, one-way ANOVA was performed. After statistically significant differences were determined, a post-hoc pairwise comparison was performed using Dunnett's test, in which vehicle control groups were compared to either PFE or SOY groups. When the results of Bartlett's test were significant, treated and control groups was compared using the KruskalWallis test and non-parametric type Dunnett's test. For comparisons between vehicle and positive control groups, the body and uterus weights were initially analyzed using the F-test. When the results of the F-test were not significant, a student's $t$-test was performed. However, when the results of the F-test were determined to be significant, the Aspin-Welch's $t$-test was subsequently performed.

\section{Results}

\subsection{ER-Binding Assay}

The ER-binding curves for the 5 compounds are shown in Figure 2. Table 1 lists the $\mathrm{EC}_{50}$ values of each chemical and their relative binding affinities. The binding affinities for ER- $\alpha$ and $-\beta$ were observed in the following order; $\beta$-estradiol $>$ genistein $>$ equol $>$ tectorigenin $>6$ hydroxygenistein. The relative binding affinities of tectorigenin for ER- $\alpha$ and $-\beta$ were 0.04 and 0.02 that of genistein, respectively, and the relative binding affinity of 6-hydroxygenistein to ER- $\alpha$ was 0.01 that of genistein. However, an $\mathrm{EC}_{50}$ value for 6-hydroxygenistein could not be determined with ER- $\beta$.

\subsection{Uterotrophic Bioassays}

There were no significant differences in the final body weight among the groups (data not shown).

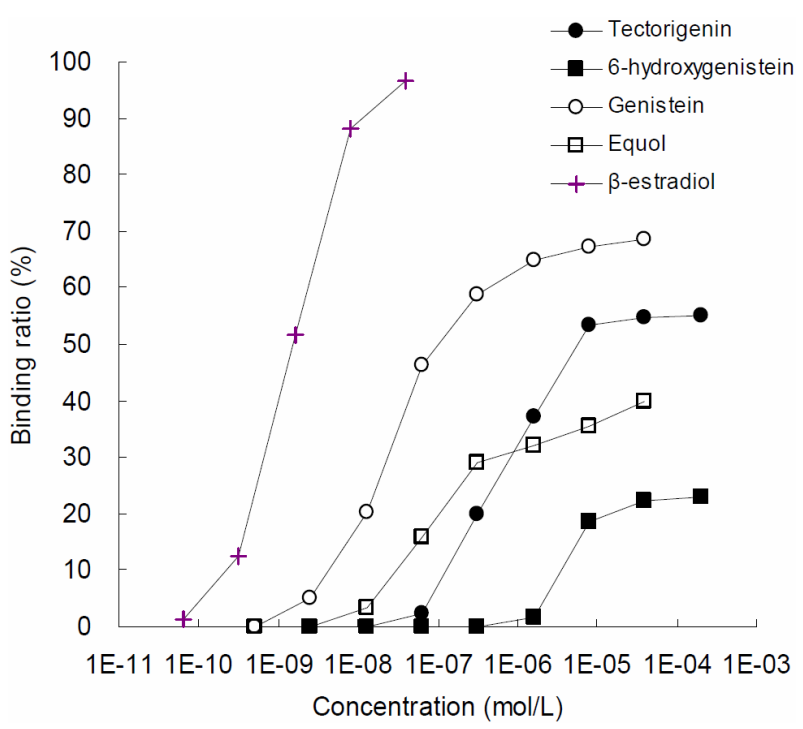

(a) ER- $\alpha$

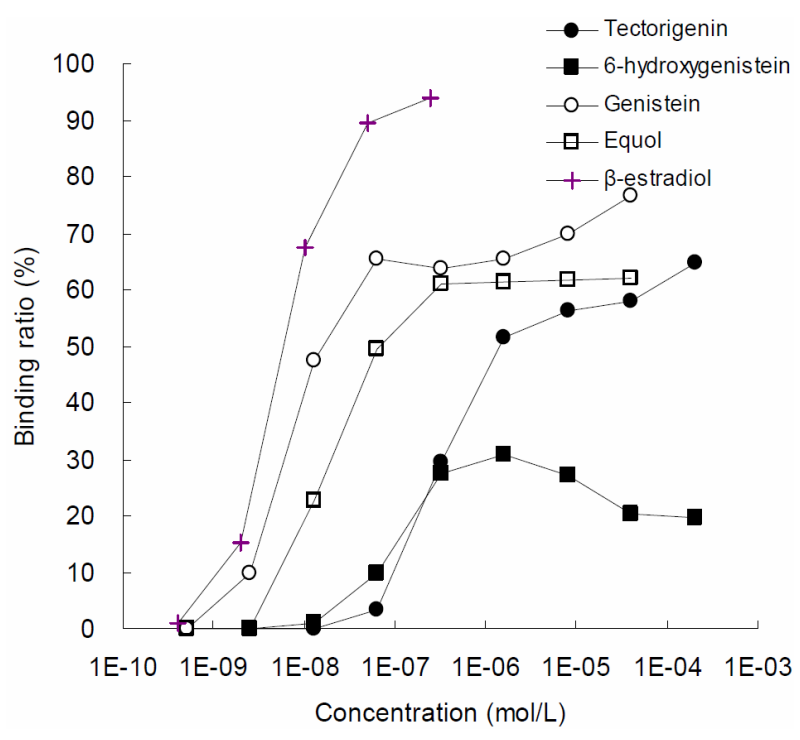

(b) ER- $\beta$

Figure 2. The ER-binding curves for the test substrates. 
In the both SOY groups (500 mg/kg and $1000 \mathrm{mg} / \mathrm{kg}$ ), the wet and blotted uterus weights were significantly higher than those of the control group (Table 2). Additionally, macroscopic examination revealed that the thickening of the uterine wall was observed in SOY groups $(500 \mathrm{mg} / \mathrm{kg}$ and $1000 \mathrm{mg} / \mathrm{kg}$ : Table 3). On the other hand, no abnormalities were observed in the uterine weights and macroscopic examination of PFE groups $(250 \mathrm{mg} / \mathrm{kg}, 500 \mathrm{mg} / \mathrm{kg}$, and $1000 \mathrm{mg} / \mathrm{kg}$ ).

\section{Discussion}

The results of the uterotrophic bioassays demonstrate that PFE cannot cause adverse estrogenic effects, even at a dose of $1000 \mathrm{mg} / \mathrm{kg} / \mathrm{day}$, which is the limit dose designated in the current draft protocol by the OECD. On the other hand, SOY appears to cause adverse estrogenic effects when excessively consumed.
In this study, the non-observed adverse effect level (NOAEL) of SOY was considered to be $250 \mathrm{mg} / \mathrm{kg}$ (equivalent to $107 \mathrm{mg} / \mathrm{kg}$ soy isoflavones), and the lowest observed adverse effect level (LOAEL) was also considered to be $500 \mathrm{mg} / \mathrm{kg}$ in uterotrophic bioassays (equivalent to $214 \mathrm{mg} / \mathrm{kg}$ soy isoflavones). The soy isoflavone genistein was reported to induce an increase in uterine wet weight at a dose of $100 \mathrm{mg} / \mathrm{kg}$ [16]. The test substrate used in the present study was a soy isoflavone mixture, which contained genistein, daidzein, and glycitein. The binding affinities of daidzein to ER- $\alpha$ and $-\beta$ was 0.27 and 0.03 that of genistein, respectively. In addition, the binding affinities of glycitein to ER- $\alpha$ and $-\beta$ were 0.15 and 0.03 that of genistein, respectively [14]. Notably, genistein exhibited the highest binding affinities for ERs compared to those of other soy isoflavones. Consequently, genistein can potentially induce increases in uterine wet weight at comparatively low dosages.

Table 1. Binding affinities of test substrates for human estrogen receptors- $\alpha$ and $-\beta$.

\begin{tabular}{ccccccc}
\hline & \multicolumn{2}{c}{$\mathrm{EC}_{50}$} & \multicolumn{4}{c}{ Relative binding affinity } \\
\cline { 2 - 7 } & $\mathrm{ER}-\alpha$ & $\mathrm{ER}-\beta$ & \multicolumn{2}{c}{ vs $\beta$-Estradiol } & \multicolumn{2}{c}{ vs Genistein } \\
\cline { 2 - 7 } & $(\mathrm{mol} / \mathrm{L})$ & $(\mathrm{mol} / \mathrm{L})$ & $\mathrm{ER}-\alpha$ & $\mathrm{ER}-\beta$ & $\mathrm{ER}-\alpha$ & ER- $\beta$ \\
\hline$\beta$-Estradiol & $1.5 \times 10^{-9}$ & $5.5 \times 10^{-9}$ & 100.0 & 100.0 & 186.7 & 136.4 \\
Genistein & $2.8 \times 10^{-8}$ & $7.5 \times 10^{-9}$ & 5.4 & 73.3 & 100.0 & 100.0 \\
Equol & $8.9 \times 10^{-8}$ & $19 \times 10^{-9}$ & 1.7 & 28.9 & 31.5 & 2.5 \\
Tectorigenin & $65 \times 10^{-8}$ & $340 \times 10^{-9}$ & 0.2 & 1.6 & 4.3 & 2.2 \\
6-Hydroxygenistein & $200 \times 10^{-8}$ & $\mathrm{NC}$ & 0.1 & $\mathrm{NC}$ & 1.4 & $\mathrm{NC}$ \\
\hline
\end{tabular}

Table 2. Results of absolute uterus weight.

\begin{tabular}{|c|c|c|c|c|c|}
\hline & & \multicolumn{2}{|c|}{ Wet uterus (mg) } & \multicolumn{2}{|c|}{ Blotted uterus (mg) } \\
\hline & Control & $82.5 \pm 9.6$ & & $81.2 \pm 9.3$ & \\
\hline \multirow[t]{3}{*}{ PFE } & $250 \mathrm{mg} / \mathrm{kg}$ & $84.4 \pm 24.5$ & & $82.8 \pm 23.2$ & \\
\hline & $500 \mathrm{mg} / \mathrm{kg}$ & $81.4 \pm 7.4$ & & $80.4 \pm 7.5$ & \\
\hline & $1000 \mathrm{mg} / \mathrm{kg}$ & $90.5 \pm 16.5$ & & $89.3 \pm 16.7$ & \\
\hline \multirow[t]{3}{*}{ SOY } & $250 \mathrm{mg} / \mathrm{kg}$ & $92.8 \pm 11.0$ & & $91.6 \pm 11.0$ & \\
\hline & $500 \mathrm{mg} / \mathrm{kg}$ & $116.3 \pm 18.9$ & ${ }^{* *}$ & $114.7 \pm 18.5$ & ** \\
\hline & $1000 \mathrm{mg} / \mathrm{kg}$ & $160.2 \pm 20.8$ & ${ }^{* *}$ & $156.0 \pm 21.7$ & ** \\
\hline $\mathrm{EE}$ & $0.6 \mu \mathrm{g} / \mathrm{kg}$ (s.c.) & $321.8 \pm 31.2$ & $* *$ & $305.0 \pm 22.7$ & ** \\
\hline
\end{tabular}

Data are expressed as the mean $\pm \mathrm{SD}$. The values marked with asterisks differ significantly from the control group $\left({ }^{* *} p<0.01\right)$.

Table 3. Summary of macroscopic examination.

\begin{tabular}{|c|c|c|c|c|c|c|c|c|}
\hline & \multirow{2}{*}{ Control } & \multicolumn{3}{|c|}{ PFE } & \multicolumn{3}{|c|}{ SOY } & \multirow{2}{*}{$\frac{\mathrm{EE}}{0.6 \mu \mathrm{g} / \mathrm{kg} \text { (s.c.) }}$} \\
\hline & & $250 \mathrm{mg} / \mathrm{kg}$ & $500 \mathrm{mg} / \mathrm{kg}$ & $1000 \mathrm{mg} / \mathrm{kg}$ & $250 \mathrm{mg} / \mathrm{kg}$ & $500 \mathrm{mg} / \mathrm{kg}$ & $1000 \mathrm{mg} / \mathrm{kg}$ & \\
\hline No abnormalities & 6 & 6 & 6 & 6 & 6 & 5 & 0 & 0 \\
\hline \multicolumn{9}{|l|}{ Uterus } \\
\hline Thickening of wall & 0 & 0 & 0 & 0 & 0 & 1 & 6 & 6 \\
\hline \multicolumn{9}{|l|}{ Vagina } \\
\hline Retention of mucus in vaginal cavity & 0 & 0 & 0 & 0 & 0 & 0 & 0 & 1 \\
\hline
\end{tabular}


The respective binding affinities of the aglycone forms of the major isoflavones in PFE, tectorigenin and 6-hydroxygenistein, for ERs were the following: ER- $\alpha, 0.04$ and 0.01 that of genistein; and ER- $\beta, 0.02$ and 0.00 that of genistein, respectively. The binding affinities of daidzein, glycitein, biochanin A, and formononetin for ER- $\alpha$ were reported to be $0.27,0.15,0.16,0.03$ those of genistein, respectively, and the binding affinities of daidzein, glycitein, biochanin $\mathrm{A}$, formononetin for $\mathrm{ER}-\beta$ were 0.03 , $0.03,0.01,0.02$ those of genistein, respectively [14]. The relative binding affinities of genistein to the ER- $\alpha$ and $-\beta$ in the present study were similar to the values previously reported. Briefly, tectorigenin and 6-hydroxygenistein have lower ER binding affinities compared to those of daidzein and biochanin A. It is reported that daidzein and biochanin A did not induce an increase in uterine wet weight at doses within the range of $0.00001-1000$ $\mathrm{mg} / \mathrm{kg}$ in uterotropic bioassays [16]. In the present study, PFE did not increase the uterine weight at a dose of 1000 $\mathrm{mg} / \mathrm{kg}$, because the major isoflavones in PFE only exhibit slight estrogenic activity.

Moreover, the present study suggests that PFE does not contain compounds that exhibit strong estrogenic activity. The legume plant Pueraria mirifica has been reported to contain miroestrol and deoxymiroestrol [17], and the extent of the estrogenic activity of these compounds are approximately 4 and 20 times higher than that of genistein [18]. In particular, miroestrol promotes uterine and vaginal growth in the immature female mice [19], and Pueraria mirifica influences menstrual cycles and suppresses ovulation at doses of $10 \mathrm{mg} / \mathrm{kg}$ and $100 \mathrm{mg} /$ day in female monkeys [20]. Therefore, if PFE contains compounds that exhibit strong estrogenic effects, such as miroestrol and deoxymiroestrol, PFE would have to be administered at considerably low dosages to cause an increase in uterine weight. In fact, we performed a comprehensive mass analysis of the components compriseng PFE and miroestrol and deoxymiroestrol were not detected (unpublished data). Thus, our results confirm the safety of PFE with regards to its estrogenic activity.

In conclusion, tectorigenin and 6-hydroxygenistein, the major isoflavones of PFE exhibit weak estrogenic effects. Therefore, PFE does not have the potential to cause adverse estrogenic effects in vivo and lacks compounds that exhibit strong estrogenic activities. The results of this study further confirms the safety of PFE as a food supplement.

\section{Acknowledgements}

The authors are grateful to SEKISUI MEDICAL Co. Ltd. and Chemicals Evaluation and Research Institute for performing ER-binding assays and the uterotrophic bioassays.

\section{REFERENCES}

[1] Q. Chen, L. Wang and S. Ye, "The Study of Anti-Inflammatory and Anti-Oxidant Effects of the Five Edible Plants," Journal of Medicinal Plants Research, Vol. 6, No. 17, 2012, pp. 3351-3358.

http://www.academicjournals.org/jmpr/pdf/pdf2012/9Ma y/Chen\%20et\%20al.pdf

[2] T. Kamiya, Y. Matsuzuka, N. Kusaba, M. Ikeguchi, K. Takagaki and K. Kondo, "Preliminary Reseach for the Anti-Obesity Effect of Puerariae Flos Extract in $\mathrm{Hu}-$ mans," Journal of Health Science, Vol. 57, No. 6, 2011, pp. 521-531. doi:10.1248/jhs.57.521

[3] T. Kamiya, A. Takano, Y. Matsuzuka, N. Kusaba, M. Ikeguchi, K. Takagaki and K. Kondo, "Consumption of Pueraria Flower Extract Reduces Body Mass Index via a Decrease in the Visceral Fat Area in Obese Humans," Bioscience, Biotechnology, and Biochemistry, Vol. 76, No. 8, 2012, pp. 1511-1517. doi:10.1271/bbb.120235

[4] T. Kamiya, M. Sameshima-Kamiya, R. Nagamine, M. Tsubata, M. Ikeguchi, K. Takagaki, T. Shimada and M. Aburada, "The Crude Extract of Puerariae Flower Exerts Antiobesity and Antifatty Liver Effects in High-Fat DietInduced Obese Mice," Evidence-Based Complementary and Alternative Medicine, 2012, Article ID: 272710. http://www.ncbi.nlm.nih.gov/pmc/articles/PMC3368216/

[5] T. Kamiya, R. Nagamine, M. Sameshima-Kamiya, M. Tsubata, M. Ikeguchi and K. Takagaki, "The Isoflavone-Rich Fraction of the Crude Extract from Puerariae Flower Increases Oxygen Consumption and BAT UCP1 Expression in High-Fat Diet-Fed Mice," Global Journal of Health Science, Vol. 4, No. 5, 2012, pp. 147-155. doi:10.5539/gihs.v4n5p147

[6] L. Rowland, M. Faughnan, L. Hoey, K. Wähälä, G. Williamson and A. Cassidy, "Bioavailability of Phyto-Oestrogens," The British Journal of Nutrition, Vol. 89, No. 1, 2003, pp. S45-S58.

[7] K. Hiramaya, Y. Matsuzuka, T. Kamiya, M. Ikeguchi, K. Takagaki and K. Itoh, "Metabolism of Isoflavones Found in the Pueraria thomsonii Flower by Human Intestinal Microbiota," Bioscience Microflora, Vol. 30, No. 4, 2011, pp. 135-140.

[8] R. Tsuchihasi, M. Kodera, S. Sakamoto, Y. Nakajima, T. Yamazaki, Y. Niiho, T. Nohara and J. Kinjo, "Microbial Transformation and Bioactivation of Isoflavones from Pueraria Flowers by Human Intestinal Bacterial Strains," Journal of Natural Medicine, Vol. 63, No. 3, 2009, pp. 254-260. doi:10.1007/s11418-009-0322-Z

[9] M. S. Anthony, T. B. Clarkson, C. L. Hughes, T. M. Morgan and G. L. Burke, "Soybean Isoflavones Improve Cardiovascular Risk Factors without Affecting the Reproductive System of Peripubertal Rhesus Monkeys," The Journal of Nutrition, Vol. 126, No. 1, 1996, pp. 43-50.

[10] J. J. Anderson, W. W. Ambrose and S. C. Garner, "Biphasic Effects of Genistein on Bone Tissue in the Ovariectomized, Lactating Rat Model," Proceedings of the Society for Experimental Biology and Medicine, Vol. 217, No. 3, 1998, pp. 345-350.

[11] Y. H. Ju, C. D. Allred, K. F. Allred, K. L. Karko, D. R. 
Doerge and W. G. Helferich, "Physiological Concentrations of Dietary Genistein Dose-Dependently Stimulate Growth of Estrogen-Dependent Human Breast Cancer (MCF-7) Tumors Implanted in Athymic Nude Mice," The Journal of Nutrition, Vol. 131, No. 11, 2001, pp. 29572962.

[12] L. J. Lu, K. E. Anderson, J. J. Grady, F. Kohen and M. Nagamani, "Decreased Ovarian Hormones during a Soya Diet: Implications for Breast Cancer Prevention," Cancer Research, Vol. 60, No. 15, 2000, pp. 4112-4121.

[13] J. Kanno, L. Onyon, J. Haseman, P. Fenner-Crisp, J. Ashby and W. Owens, "The OECD Program to Validate the Rat Uterotrophic Bioassay to Screen Compounds for in Vivo Estrogenic Responses: Phase 1," Environmental Health Perspectives, Vol. 109, No. 8, 2001, pp.785-794. doi:10.1289/ehp.01109785

[14] S. Y. Choi, T. Y. Ha, J. Y. Ahn, S. R. Kim, K. S. Kang, I. K. Hwang and S. Kim, "Estrogenic Activities of Isoflavones and Flavones and Their Structure-Activitiy Relationships," Planta Medica, Vol. 74, No. 1, 2007, pp. 2532. doi:10.1055/s-2007-993760

[15] T. Zacharewski, "Identification and Assesement of Endocrine Disruptors: Limitations of in Vivo and in Vitro Assays," Environmental Health Perspectives, Vol. 106, No. 2, 1998, pp. 577-582. doi:10.1289/ehp.98106577

[16] W. N. Jefferson, E. Padilla-Banks, G. Clark and R. R. Newbold, "Assesing Estrogenic Activity of Phytochemi- cals Using Transcriptional Activation and Immature Mouse Uterotrophic Responses," Journal of Chromatography B. Analytical Technologies in the Biomedical and Life Science, Vol. 777, No. 1-2, 2002, pp. 179-189.

[17] S. Chansakaow, T. Ishikawa, H. Seki, K. Sekine-Yoshizawa, M. Okada and C. Chaichantipyuth, "Identification of Deoxymiroesterol as the Actual Rejuvenating Principle of "Kwao Keur", Pueraria Mirifica. The Known Miroesterol May Be an Artifact," Journal of Natural Products, Vol. 63, No. 2, 2000, pp. 173-175. doi:10.1021/np990547v

[18] A. Matumura, A. Ghosh, G. S. Pope and P. D. Darbre, "Comparative Study of Oestrogenic Properties of Eight Phytoestrogens in MCF7 Human Breast Cancer Cells," The Journal of Steroid Biochemistry and Molecular Biology, Vol. 94, No. 5, 2005, pp. 431-443. doi:10.1016/j.jsbmb.2004.12.041

[19] H. E. Jones and G. S. Pope, "A Study of the Action of Miroestrol and Other Oestrogens on the Reproductive Tract of the Immature Female Mouse," The Journal of Endocrinology, Vol. 20, 1960, pp. 229-235. doi:10.1677/joe.0.0200229

[20] H. Trisomboon, S. Malaivijitnond, G. Watanabe and K. Taya, "Ovulation Block by Pueraria Mirifica: A Study of Its Endocrinological Effect in Female Monkeys," Endocrine, Vol. 26, No. 1, 2005, pp. 33-39. doi:10.1385/ENDO:26:1:033 